\title{
A Comparative Evaluation of the Performance of the multi-hop IoB-DTN routing protocol
}

\author{
Yosra ZGUIRA ${ }^{1,2}$, Hervé RIVANO ${ }^{1}$, and Aref MEDDEB ${ }^{2}$ \\ 1 Univ Lyon, INSA Lyon, Inria, CITI F-69621 Villeurbanne, France \\ 2 NOCCS Laboratory Higher Institute of Computer Science and Communication Technologies, \\ ISITCom, University of Sousse Sahloul, 4054, Tunisia
}

\begin{abstract}
Following the trend of the Internet of Thing, public transport systems are seen as an efficient bearer of mobile devices to generate and collect data in urban environments. Bicycle sharing system is one part of the city's larger transport system. In this article, we study the "Internet of Bikes" IoB-DTN protocol which applies the Delay Tolerant Network (DTN) paradigm to the Internet of Things (IoT) applications running on urban bike sharing system based sensor network. We evaluate the performances of the protocol with respect to the transmission power. Performances are measured in terms of delivery rate, delivery delay, throughput and energy cost. We also compare the multi-hop IoB-DTN protocol to a low-power wide-area network (LPWAN) technology. LPWAN have been designed to provide cost-effective wide area connectivity for small throughput IoT applications: multiyear lifetime and multikilometer range for battery-operated mobile devices. This work aims at providing network designers and managers insights on the most relevant technology for their urban applications that could run on bike sharing systems. To the best of our knowledge, this work is the first to provide a detailed performance comparison between multi-hop and long range DTN-like protocol being applied to mobile network IoT devices running a data collection applications in an urban environment.
\end{abstract}

\section{Introduction}

The self-service bicycles, also known as bicycle-sharing systems, have been introduced as part of the urban transportation system in many cities of the world. They allow people to borrow a bicycle from bike station A and return it at bike station B with a very small price. As of June 2014, public bicycle sharing systems were available in 50 countries on 5 continents, including 712 cities and having approximately 806,200 bicycles at 37,500 stations [1]. The present paper focuses on the use of IoT on real networks and in particular on connected bikes. We are interested in opportunistic communications based on converge cast algorithm. We consider a smart bike sharing system to collect and transfer the data from bikes to a set of sinks. In our preceding work [2], we proposed the "Internet of Bikes" IoB-DTN protocol which applies the DTN paradigm to the IoT applications running on urban bike sharing system based sensor network. The Delay Tolerant Networking approach is designed for intermittent connections resulting in a lack of instantaneous end-to-end paths between mobile devices [3]. In this type of network, routing is performed over time to send data by employing long-term storage 
at the intermediate nodes. Thus, the intermediate node stores the receiving data, carries it while a contact is not available and forwards when the connection occurs. Therefore, data will be relayed hop by hop until reaching its destination.

In this article, we give a more detailed performance evaluation of IoB-DTN protocol. First, we evaluate the performance of the protocol by ranging the transmission power. Performance is measured in terms of delivery rate, delivery delay, throughput and protocol cost resulting in the number of packets transmitted and received in the network. Then, we compare the performance of the multi-hop IoB-DTN protocol with a low-power wide-area network (LPWAN) technology. The performance metrics used for the comparison are the energy consumption and the throughput. The low power wide area networks represent a novel communication paradigm in the evolution of the wireless communication technologies [4]. They have been designed to provide cost-effective wide area connectivity for the IoT applications: multiyear lifetime and multikilometer range for battery-operated mobile devices. The battery lifetimes can possibly operate up to ten years and the operating range is from over $10 \mathrm{~km}$ in rural areas up to $1-2 \mathrm{~km}$ in urban environments. LPWANs consume low power and use a low data rate for data transmission. They are typically seen as cellular networks by connecting end devices (ED) directly to base stations (BS) which relay data packets between the EDs and an application server. An ED communicates only to a BS forming a star-topology network that brings huge energy saving advantages. LPWAN technologies include unlicensed band technologies (e.g. Sigfox, LoRa/LoRaWAN, and Weightless), advanced cellular technologies (e.g. LTEM and NB-IoT) and recent reforms to IEEE standards (e.g. IEEE 802.11ah, IEEE 802.15.4g, and IEEE 802.15.4k). In this paper, we compare IoB-DTN protocol to LoRa/LoRaWAN ${ }^{3}$ technology which is based on chirp spread spectrum modulation. The use of this modulation provides enhanced performances in terms of range, significantly increasing the robustness of the signal and the sensitivity of the receiver while maintaining low power consumption.

Our contributions can be summarized as follows:

- A performance evaluation of IoB-DTN protocol for different transmission power of sensors. The optimal value is conducted in terms of the performance metrics mentioned before.

- A performance comparison between the multi-hop IoB-DTN protocol and the long range LoRa technology.

- Results are discussed to identify the best technology to adapt in IoT data collection applications running on urban bike sharing system.

The rest of the article is organized as follows. The following section discusses the related work. The description of our scenario is presented in Section 3. The simulation environment is described in Section 4. The performance evaluations of IoB-DTN protocol as well as the analysis of our results are discussed in Section 5 and Section 6 respectively. Finally, Section 7 concludes the paper.

\footnotetext{
${ }^{3}$ LoRa: https://www.lora-alliance.org/
} 


\section{Related work}

In the literature, several researchers have focused on communication based public transport networks. For examaple, the DakNet [5] provides low-cost digital communication to remote villages in India and Cambodia. Buses are used in DakNet to transfer data between Internet access points and Internet kiosks in villages. The KioskNet [6] uses buses and cars as "mechanical backhaul" devices to transfer data between remote villages and Internet gateway. Zhao et al. [7] present a vehicle assisted data delivery (VADD) protocol for vehicular ad hoc networks. The VADD is based on the carry and forward paradigm where vehicles are used as data carriers and the path to the destination is determined based on the ad hoc connectivity of the vehicles.

Bikes are considered also as an urban transport system to sense, collect and forward data. The BikeNet project [8] corresponds to the earliest working mobile sensing and sparse radio network connectivity system for cyclists. In BikeNet, the sensors are embedded into a cyclist's bike to collect quantitative data about the cyclist's journeys. The data gathered is forwarded by the cellular data channel of the cyclist's mobile phone. Nakamura et al. [9] propose the design of a web framework for a ubiquitous sensor network (u-framework). In the experimental field trial, they used bikes equipped with small and high-precision NO2 sensors to gather and share information on air pollution in Tokyo, Japan.

Over the past few years, many works focused on applying DTN paradigm to the Internet of Things. Wirtz et al. [10] discuss the notion of a "Challenged IoT" under intermittent Internet connectivity. They discuss the need to enable interaction between smart objects and mobile users in the Internet of Things. They propose Direct Interaction with Smart Challenged Objects (DISCO), enabling objects to define their interaction patterns and interface. Al-Turjman et al. [11] propose DIRSN, an optimized delay-tolerant framework for integrated RFID-sensor networks (RSNs) in the IoT. Their framework provides an optimized architecture for integrated RSNs besides to a delaytolerant routing scheme. In [12], the authors propose an enhanced architecture to interconnect standard-based machine-to-machine (M2M) platform to opportunistic networks in order to collect data from sensor devices.

Many researches focused also on applying DTN with IoT and more precisely in the field of delay-tolerant WSN that focus on routing algorithms. Most of these proposals do not use standard protocols, but they are dedicated to targeted sensors or applications, e.g. underwater sensor networks. In [2], we introduced the "Internet of Bikes" IoB-DTN protocol which applies the DTN paradigm to the IoT applications running on urban bike sharing system based sensor network. It is designed for being applied to mobile network IoT devices running a data collection application. It is a multi-hop protocol where data are forwarded via bike-to-bike communication.

In this article we aim at providing an evaluation of IoB-DTN protocol by varying the transmission power of the sensors and a comparison of IoB-DTN with a low-power wide-area network (LPWAN) technology. Several LPWAN technologies have been deployed in previous years. There are many forms of LPWA networks and they all have a different market approach and technology stack. Among them, we quote Sigfox, LoRaWAN, Weightless. The Sigfox ${ }^{4}$ technology was developed in 2010 by the start-up

\footnotetext{
${ }^{4}$ Sigfox: https://www.sigfox.com/en
} 
Sigfox in Toulouse, France. It applies a technique of the ultra-narrowband IoT communications designed to support IoT deployments over long range communications. It operates in the $869 \mathrm{MHz}$ (in Europe) and $915 \mathrm{MHz}$ (in North America) bands. LoRa was developed by the start-up Cycleo in 2009 in Grenoble, France and was purchased by Semtech (USA) in 2012. In 2015, LoRa was standardized by LoRa-Alliance and was deployed in 42 countries. It is based on chirp spread spectrum modulation which uses the same low power characteristics as FSK modulation but greatly increases the communication range. The LoRa physical layer operates on the 433 (in Asia), 868 (in Europe) or 915 (in North Ametica) MHz ISM bands. Three open standards for LPWAN were developed by the Weightless Special Interest Group: Weightless-W, Weightless-N and Weightless- $\mathrm{P}^{5}$. Weightless-W is based on narrow-band FDMA channels with Time Division Duplex between uplink and downlink. It is designed to operate in TV whitespaces $(470-790 \mathrm{MHz})$. Weightless-N is based on the ultra-narrow-band technology and it provides only uplink communication. Weightless-P provides ultra-high performance LPWAN connectivity.

\section{Scenario description}

We consider an ad-hoc network of bikes. Each bike has embedded sensors, a 802.11p communication device, periodically generates a data packet and stores it in its buffer. All bike stations are equipped with base stations which are connected to the Internet. Each bicycle station has a $802.11 \mathrm{p}$ interface and acts as a fixed sink. In IoB-DTN protocol, a packet is relayed until it reaches one of the sinks. IoB-DTN is based on the DTN paradigm which is designed for low connectivity that results in the absence of instantaneous end to end path. Due to the intermittent connection between bikes, the store-carry-forward mechanism is applied. The data are stored in the buffers of the intermediate nodes and sent at a later time to the final destination or to another intermediate nodes. The IoB-DTN protocol is inspired by flooding protocols which diffuse multiple copies of the packet in the network in order to maximize its probability to reach the destination. In particular, it is a lightweight version of Binary Spray and Wait routing protocol which limits the number of copies sprayed in the network to minimize the resource consumption. In Binary Spray and Wait protocol, the source node starts with $\mathrm{N}$ copies of the packet, and whenever it encounters a neighbor node, it sends half of copies to it and keeps the other half for itself. If it has only one copy of the packet, it switches to direct transmission to the destination node.

In IoB-DTN protocol, each node generates a packet $P$ at each reading period. The packet is then stored, with the corresponding number of copies $N$, if the buffer management policy provides a slot. When the duty cycle is over, each node verifies if there is base stations in its neighborhood. If it exists, it sends all the data packets stored in its buffer and then it receives the acknowledgements (ACKs) from the corresponding sinks. If there is only neighbouring nodes, it forwards only the packets that have more than one copy. In the packet reception phase, each node calculates the new number of copies $N^{\prime}$ that it should keep, it stores the received packet if there is a slot in the buffer and it sends an ACK to the source node. When a node receives an ACK, it verifies the sender type. If it is a base station, it deletes the packet from its buffer. If not, it updates the number of copies of the corresponding packet.

\footnotetext{
${ }^{5}$ Weightless: https://www.weightless.org/
} 
Note that the copies of a data packet stored in a buffer are virtual. We are incrementing a counter and each packet occupies only one slot of the buffer. The buffer management policy is a major parameter of IoB-DTN. When the buffer is not full, it provides the next slot. If it is full, it must decide which packet should be kept and which packet should be discarded. In our previous work [2], we have proposed and simulated four buffer management policies. From the results obtained, GPP (Generated Packet Priority) policy which protects the self production gives the best results in terms of loss rate and delivery delay. In GPP policy, when the buffer is full and a new packet is generated, it replaces the oldest received packet. If there are only generated packets stored in the buffer, it replaces the oldest one. In this strategy, all received packets are discarded. In this paper, we consider the GPP policy and the number of copies is set to 8 in all our simulations.

\section{Simulation environment}

This section describes the simulation settings used for our scenarios. The urban environment we used to evaluate our proposal is the city of Lyon, France. The bike sharing system in Lyon is called Vélo'v6. The system, launched in May 2005, provides over 3000 bicycles available from over 350 stations situated around the cities of Lyon and Villeurbanne. The bicycles can be taken from any station by citizens and returned to any other station. The platform "Data Grand Lyon" 7 provides open data including the description of the Vélo'v system. These data are integrated with the map of Lyon from OpenStreetMap ${ }^{8}$. The fusion of these two data is then imported to SUMO, an open source road traffic simulator [13]. SUMO simulates a realistic mobility of the bikes on the streets of the map. The Veins framework ${ }^{9}$ connects SUMO to the event-based network simulator OMNeT++ ${ }^{10}$ and provides realistic radio propagation and models of 802.11p. For our simulations, we assume 49 bicycle stations deployed in the city center of Lyon as depicted in Figure 1 as well as 51 bikes moving between those bike stations. The simulation time is 30 minutes. We simulate 10 scenarios with different paths of the bikes in each scenario. We simulate four sets of parameters as shown in Table 1 by varying the buffer size and the duty cycle. The latter corresponds to the period defined in seconds to send all data packets stored in the buffer.

\section{Performance evaluation of IoB by ranging the transmission power}

In this section, we evaluate the impact of IoB-DTN protocol on the energy . The transmission power used in our simulations in [2] was $10 \mathrm{~mW}$, which gives a communication range $\backsim 350$ meters. In the present paper, we focuse on the performance evaluation of IoB by assessing four values of the transmission power: $1 \mathrm{~mW}, 5 \mathrm{~mW}, 10 \mathrm{~mW}$ and $20 \mathrm{~mW}$. We present the average results of the ten simulated scenarios.

\footnotetext{
${ }^{6}$ Vélo’v: https://velov.grandlyon.com

${ }^{7}$ Data Grand Lyon: https://data.grandlyon.com

${ }^{8}$ Openstreetmap: https://www.openstreetmap.org

${ }^{9}$ Veins: http://veins.car2x.org/

${ }^{10}$ OMNeT++: https://omnetpp.org
} 


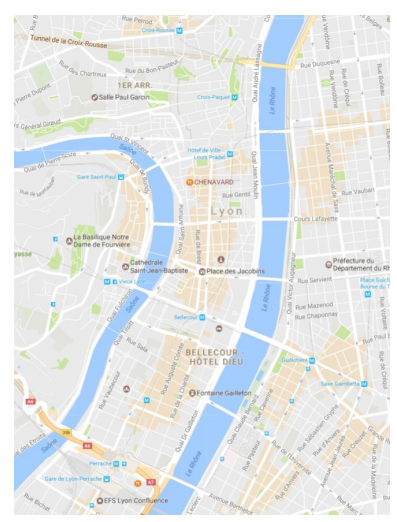

Fig. 1: Considered area of Lyon

\begin{tabular}{|l|l|l|}
\hline & Buffer size & Duty cycle (s) \\
\hline Case 1 & 250 & 50 \\
\hline Case 2 & 250 & 150 \\
\hline Case 3 & 500 & 50 \\
\hline Case 4 & 500 & 150 \\
\hline
\end{tabular}

Table 1: Simulation parameters

Figure 2 shows the average distances obtained for each transmission power and for each case. We notice that by increasing the value of the transmission power, the communication range of nodes increases respectively.

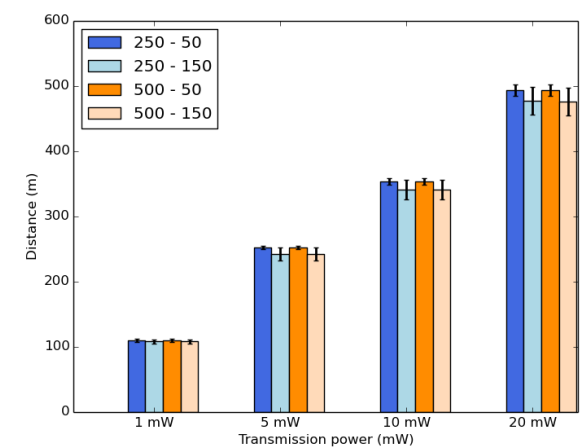

Fig. 2: Average distances

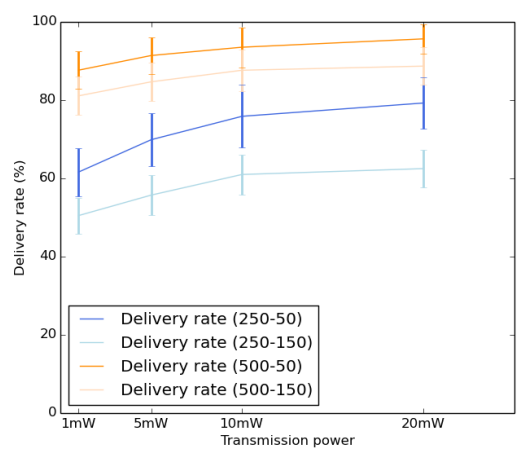

Fig. 3: Average delivery rate

The average delivery rate obtained is depicted in Figure 3. As expected the delivery rate increases by enhancing the transmission power. In this case, the communication range of bikes increases so they encounter more neighbors nodes and more base stations which allows to have a higher delivery rate.

Figure 4 shows the average delivery delays of the received packets. We notice that the impact of the transmission power on the delivery delay is negligible compared to the duty cycle and the size of the buffer. It shows that the connectivity is more impacted by the dynamics of the network than the transmission range. The size of the buffer increases the average delay partly because it prevents more packets to be discarded. The transmission range impacts the throughput of the network as depicted in Figure 5. The impact of the duty cycle and the buffer size is still very important since the whole buffer is sent at each duty cycle. 


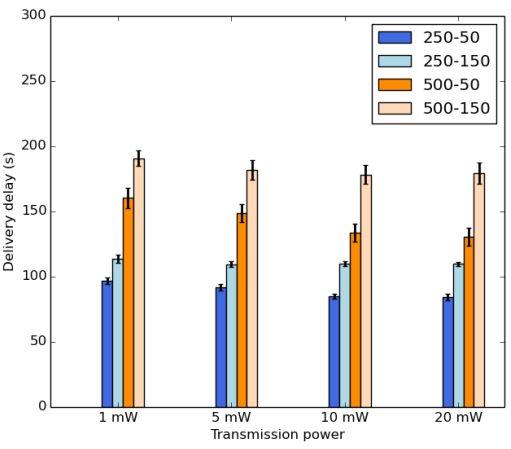

Fig. 4: Average delivery delay

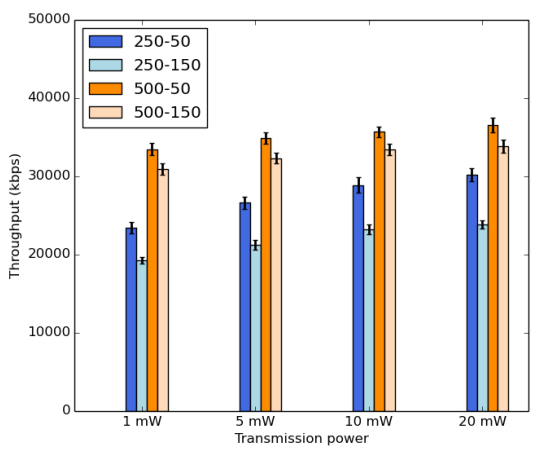

Fig. 5: Average throughput

We evaluated the average protocol cost of IoB-DTN protocol in terms of the number of transmitted and received packets in all the considered scenarios. The results illustrated in Figure 6 present all the communications between bike to bike and bike to bike station. In our setting, each bike finishing its trip forwards all the data packets stored in its buffer to the final bike station that we assume having a very high throughput and we do not consider it in energy consumption assessments. For each case, we can see two columns: the first column represents all the data packets forwarded and the second one depicts all the received data packets. On one hand, the first column contains three fields: NPSN, NASN and NPSG. First, NPSN represents the number of packets sent to nodes. Secondly, NASN is the number of acknowledgments sent to nodes. Finally, NPSG represents the number of packets sent to gateways. On the other hand, the second has two fields: NPRN and NPRG. The first one corresponds to the number of packets and acknowledgments received by nodes. The last one, NPRG, represents the number of packets received by the gateways. We notice that the average number of forwarded and received data packets increases by enhancing the transmission power. This is an obvious consequence since the communication range increases allowing more communications with neighbors bicycles or gateways. It is interesting to note that using a higher duty cycle provides a smaller protocol cost. Indeed, in this case, the packets spend more time to be stored in the buffers which decreases the communication with the remaining nodes and gateways in the network.

We notice that the increase in power and the use of a small duty cycle allow for better delivery rate, delivery delay and throughput. Whereas it leads to high energy performance. The choice of this value depends on the needs of the designers of the network. In the next section, we consider IoB-DTN with $10 \mathrm{~mW}$ as transmission power since it gives the compromise between the evaluated metrics.

\section{Performance evaluation of IoB and IoB Long-range}

In this section, we wish to compare the performance between the multi-hop IoB-DTN protocol and a long range technology. We consider IoB-DTN protocol with a radio propagation that gives us around 1 kilometer as communication range as denoted IoB- 


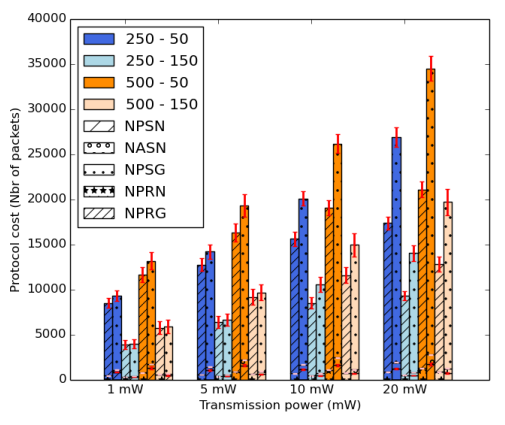

Fig. 6: Average protocol cost of IoB

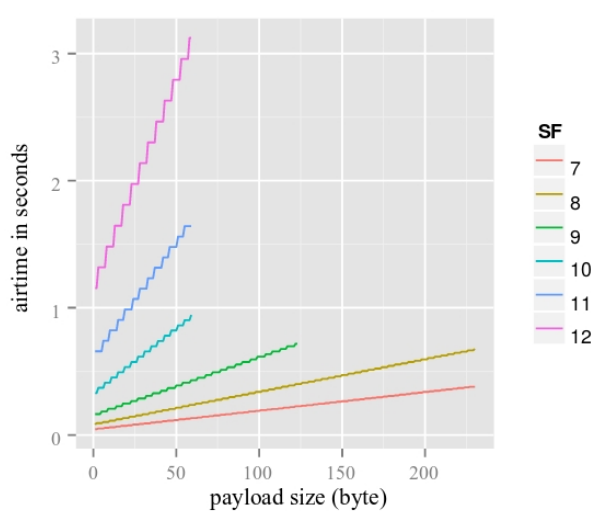

Fig. 7: Airtime for different SF and payloads [14]

LR. In IoB-LR, each node periodically generates data packets, stores them in its buffer and when the duty cycle is over and whenever a base station comes in range it sends all the data stored in its buffer. Therefore, there is only bike to bike station communication.

Since there is not a simulation model for long range technology in OMNeT++ network simulator, we assume that IoB-LR follows the Semtech SX1272 model [15] of LoRa technology. As in IoB-DTN protocol, the nodes and gateways have a $802.11 \mathrm{p}$ communication device, in our theoretical results we consider the parameters offered by the Datasheet Qualcomm AR6004 model [16], presented in Table 2.

\begin{tabular}{|l|l|l|}
\hline & $802.11 \mathrm{p}$ & LoRa \\
\hline Model & Datasheet Qualcomm AR6004 & Semtech SX1272 \\
\hline Tx & $237 \mathrm{~mA}$ & $26 \mathrm{~mA}$ \\
\hline $\mathrm{Rx}$ & $66 \mathrm{~mA}$ & $12 \mathrm{~mA}$ \\
\hline & $213 \mu \mathrm{s}$ & $250-50: 0,2 \mathrm{~s}$ \\
Packet duration & & $250-150:: 0,6 \mathrm{~s}$ \\
& & $500-50:: 0,1 \mathrm{~s}$ \\
& & $500-150: 0,3 \mathrm{~s}$ \\
\hline ACK duration & $213 \mu \mathrm{s}$ & $0.05 \mathrm{~s}$ \\
\hline & 160 Byte & $250-50: 92$ Byte \\
Packet size & & $250-150: 260$ Byte \\
& & $500-50: 20$ Byte \\
& & $500-150: 175$ Byte \\
\hline
\end{tabular}

Table 2: Parameters used

From Table 2, we remark that the packet duration of the long range technology varies according to the four cases simulated. For example, if the maximum buffer size is 250 and the duty cycle is defined as $50 \mathrm{~s}$, the time to transmit each packet to the destination is $0.2 \mathrm{~s}$. To determine the corresponding packet size of IoB-LR for each case, 
we refer the reader to Figure 7. It represents the airtime in seconds (time to transmit a packet) for different spreading factors (SF) and payloads presented by an operational LoRaWAN namely The Things Network [14]. The bandwith used is $125 \mathrm{KHz}$ and the coding rate (CR), which reports to Forward Error Correction, is $\frac{4}{5}$.

Table 3 describes the values of the payload sizes obtained for different spreading factors for each case. The spreading factor denotes the number of chirps used to encode a bit and it varies from SF7 to SF12.

Higher chirp rate allows a better reconstruction of the received signal, whereas it delays the time to send a bit. In our simulations, we consider the payload size of IoBLR using the spreading factor SF7 since it offers the highest values of payload size.

\begin{tabular}{|l|l|l|l|l|l|l|l|}
\hline & $\begin{array}{l}\text { Airtime } \\
\text { (s) }\end{array}$ & $\begin{array}{l}\text { PS (Byte) } \\
\text { SF 7 }\end{array}$ & $\begin{array}{l}\text { PS (Byte) } \\
\text { SF 8 }\end{array}$ & $\begin{array}{l}\text { PS (Byte) } \\
\text { SF 9 }\end{array}$ & $\begin{array}{l}\text { PS (Byte) } \\
\text { SF 10 }\end{array}$ & $\begin{array}{l}\text { PS (Byte) } \\
\text { SF 11 }\end{array}$ & $\begin{array}{l}\text { PS (Byte) } \\
\text { SF 12 }\end{array}$ \\
\hline $250-50$ & 0.2 & 92 & 40 & x & x & x & x \\
\hline $250-150$ & 0.6 & 260 & 220 & 100 & 28 & x & x \\
\hline $500-50$ & 0.1 & 20 & x & x & x & x & x \\
\hline $500-150$ & 0.3 & 175 & 80 & 25 & x & x & x \\
\hline
\end{tabular}

Table 3: Different payload size (PS) with different SF of IoB-LR

In our results, we evaluate the energy consumption and the throughput for IoB and IoB-LR. To evaluate the average energy consumption, we calculated the average transmission cost per bike and the average consumption background per bike. Figure 8 shows the average transmission cost per bike. It is measured in $\mathrm{mAh}$ and computed as follows:

$$
T C=[N P S * T x * D P]+[N A R *(T x+R x) * D A]+\underbrace{[N P R * R x * D P]}_{\text {for IoB }}
$$

TC is the transmit cost expressed in mAh. NPS corresponds to the number of sent packets. Tx and Rx represents the transmit and the receive consumption respectively. DP and DA are the packet and the ACK duration in seconds. NAR corresponds to the number of received ACK and NPR is the number of received packets from nodes.

We notice that IoB-LR has a higher forwarding cost because bikes send all the data packets only to the gateways. On the other hand, IoB offers a smaller forwarding cost thanks to the bike to bike communication that decreases the transmission cost in the network. In order to respect the duty cycle of radio devices regulated in Europe by section 7.2.3 of the ETSI EN300.220 standard, we consider the maximum theoretical duty cycle allowed which is $10 \%$ using the sub-bands $(869.4869 .65 \mathrm{MHz})$ [17]. The duty cycle indicates the real period during which a resource is active. Therefore, we present, in Figure 8, the average transmission cost for LoRa, respecting the maximum theoretical duty cycle $10 \%$. In fact, from the four simulated cases, the third case that has a buffer size equal to 500 slots and a time to send all the stored packets equal to 50 $\mathrm{s}$ represents the real value of the maximum duty cycle allowed by the long range LoRa technology. To fill the $\frac{1}{10}$ of 500 slots in $50 \mathrm{~s}$, the duty cycle is then $10 \%$. By following 
the same concept, the duty cycle for the four simulated cases are: $20 \%$ for the first case $250-50,60 \%$ for the sond case $250-150$ and $30 \%$ for the last case $500-150$.

We note that similar to using the maximum real duty cycle of LoRa technology, IoB gives smaller transmission cost per bike thanks to the bike to bike communication.

Figure 9 shows the average consumption background per bicycle. It corresponds to the total consumption per bike and it is measured in mAh. It is computed as follows:

For IoB:

$$
B C=\sum(T a-T d) * R x
$$

For IoB-LR:

$$
B C=\frac{R x * B W T}{D C} * \sum(T a-T d)
$$

$\mathrm{BC}$ is the average backroung consumption calulated. Ta and Td represent the arrival and the departure time of the bike expressed in seconds. DC is the duty cycle defined in seconds to send all data packets stored in the buffer. It takes as value $50 \mathrm{~s}$ or 150 $\mathrm{s}$ depending on the simulated case. BWT corresponds to the beacon waiting period defined in seconds. This period is fixed to $10 \mathrm{~s}$ in all our simulations. We notice that IoB has higher results in terms of consumption background than IoB-LR since opportunistic communications require the nodes to be always listening for beacons to relay the data packets in the network. Whereas, in long range technology, the nodes enter in sleep mode and they wake up few seconds before starting the packets forwarding. In Figure 9, we also present an optimal average consumption background per bicycle for IoB. In this case, it behaves like IoB-LR. We consider that each bike, having a full buffer, enters in sleep mode. It wakes up a few moments before the packets transmission. From the results obtained in Figure 8 and Figure 9, we can remark that IoB-LR offers lower energy consumption than the multi-hop IoB protocol.

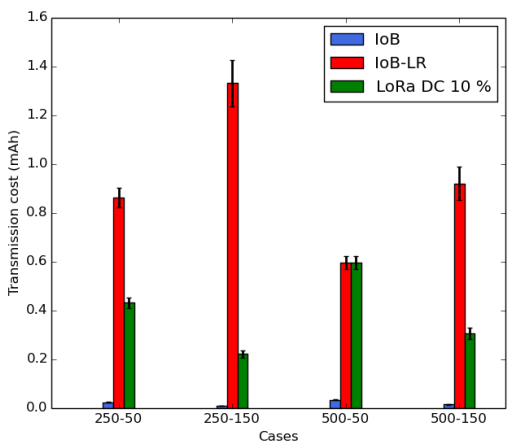

Fig. 8: Average transmission cost per bike

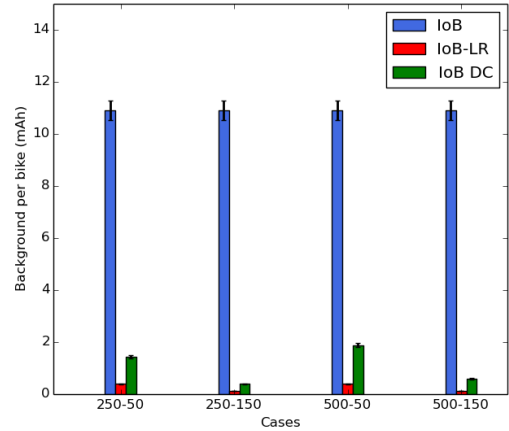

Fig. 9: Average consumption background per bike

Figure 10 shows the average throughput for IoB and IoB-LR. We note that, in all cases, IoB gives better results in terms of throughput by using a smaller duty cycle. While IoB-LR offers better throughput by adopting a higher duty cycle. 
This is related to the size of the sent packets in each simulated case. The Figure 10 shows also the throughput results for IoB-LR when respecting the maximum theoretical duty cycle allowed by LoRa technology. We remark that IoB has better throughput, in all scenarios, than the long range technology respecting the theoretical duty cycle.

In Figure 10, we also present the average throughput results for IoB-LR when respecting the effective duty cycle. It represents the real duty cycle for the bikes in each simulation scenario. It is presented in Figure 11 and it is measured as follows:

$$
E D C=\frac{N P S * R t}{\sum(T a-T d)}
$$

EDC is the effective duty cycle calculated. NPS represents the number of sent packets. Rt is the airtime defined in seconds. For example, by respecting the theoretical duty cycle for the first case simulated when having a buffer size equal to 250 slots and the period to send the packets in the buffer equal to $50 \mathrm{~s}$, the duty cycle should be $20 \%$. Whereas in reality, the effective duty cycle for this case is around $16 \%$. It is then interesting to note that IoB protocol offers better throughput, in all cases, than the long range technology respecting the theoretical and the real duty cycle.

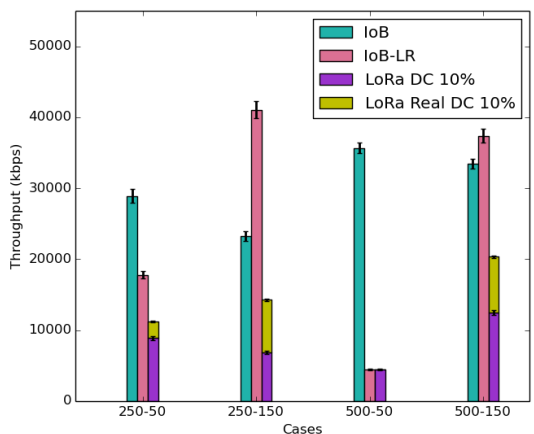

Fig. 10: Average throughput

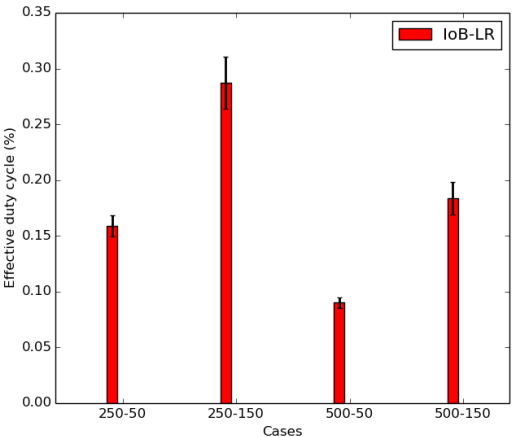

Fig. 11: Average effective duty cycle

\section{Conclusions}

In this paper, we provide two performance evaluations of IoB-DTN protocol. First, we give a performance evaluation by varying the transmission power values of sensors. This parameter is important since by increasing the sending power value the communication range of the device raises. In such case, it allows more communications with neighbors nodes and base stations which increases the delivery rate, the throughput and the energy consumption. It is worth to note that using a small duty cycle offers better delivery rate, delivery delay and throughput. Second, we provide a performance comparison of the multi-hop IoB-DTN protocol with a low-power wide-area network (LPWAN) technology, in particular LoRa/LoRaWAN. Our results show that by using a multi-hop topology, it offers better throughput while by applying a long range technology, where there is only bike to bike station communication, it gives better energy consumption. 


\section{References}

1. Susan A Shaheen, Elliot W Martin, Adam P Cohen, Nelson D Chan, and Mike Pogodzinski. Public bikesharing in north america during a period of rapid expansion: Understanding business models, industry trends \& user impacts, mti report 12-29. 2014.

2. Yosra Zguira Hervé Rivano and Aref Meddeb. IoB-DTN: a lightweight DTN protocol for mobile IoT Applications to smart bike sharing systems. WD2018, 10th IFIP Wireless Days, April 2018, Dubai, UAE.

3. Kevin Fall. A delay-tolerant network architecture for challenged internets. In Proceedings of the 2003 conference on Applications, technologies, architectures, and protocols for computer communications, pages 27-34. ACM, 2003.

4. Usman Raza, Parag Kulkarni, and Mahesh Sooriyabandara. Low power wide area networks: An overview. IEEE Communications Surveys \& Tutorials, 19(2):855-873, 2017.

5. Alex Pentland, Richard Fletcher, and Amir Hasson. Daknet: Rethinking connectivity in developing nations. Computer, 37(1):78-83, 2004.

6. Aaditeshwar Seth, Darcy Kroeker, Matei Zaharia, Shimin Guo, and Srinivasan Keshav. Lowcost communication for rural internet kiosks using mechanical backhaul. In Proceedings of the 12th annual international conference on Mobile computing and networking, pages 334345. ACM, 2006.

7. Jing Zhao and Guohong Cao. Vadd: Vehicle-assisted data delivery in vehicular ad hoc networks. IEEE transactions on vehicular technology, 57(3):1910-1922, 2008.

8. Shane B Eisenman, Emiliano Miluzzo, Nicholas D Lane, Ronald A Peterson, Gahng-Seop Ahn, and Andrew T Campbell. Bikenet: A mobile sensing system for cyclist experience mapping. ACM Transactions on Sensor Networks (TOSN), 6(1):6, 2009.

9. Takayuki Nakamura, Yukio Kikuya, Yutaka Arakawa, Motonori Nakamura, Yuka Higashijima, Yasuko Yamada Maruo, and Masayuki Nakamura. Proposal of web framework for ubiquitous sensor network and its trial application using no2 sensor mounted on bicycle. In Applications and the Internet (SAINT), 2012 IEEE/IPSJ 12th International Symposium on, pages 83-90. IEEE, 2012.

10. Hanno Wirtz, Jan Rüth, Martin Serror, Jó Ágila Bitsch Link, and Klaus Wehrle. Opportunistic interaction in the challenged internet of things. In Proceedings of the 9th ACM MobiCom workshop on Challenged networks, pages 7-12. ACM, 2014.

11. Fadi M Al-Turjman, Ashraf E Al-Fagih, Waleed M Alsalih, and Hossam S Hassanein. A delay-tolerant framework for integrated rsns in iot. Computer Communications, 36(9):998 1010, 2013

12. Asma Elmangoush, Andreea Corici, Marisa Catalan, Ronald Steinke, Thomas Magedanz, and Joaquim Oller. Interconnecting standard $\mathrm{m} 2 \mathrm{~m}$ platforms to delay tolerant networks. In Future Internet of Things and Cloud (FiCloud), 2014 International Conference on, pages 258-263. IEEE, 2014.

13. Michael Behrisch, Laura Bieker, Jakob Erdmann, and Daniel Krajzewicz. Sumo-simulation of urban mobility: an overview. In Proceedings of SIMUL 2011, The Third International Conference on Advances in System Simulation. ThinkMind, 2011.

14. Norbert Blenn and Fernando Kuipers. Lorawan in the wild: Measurements from the things network. arXiv preprint arXiv:1706.03086, 2017.

15. Lluís Casals, Bernat Mir, Rafael Vidal, and Carles Gomez. Modeling the energy performance of lorawan. Sensors, 17(10):2364, 2017.

16. Qualcomm Technologies, AR6004 Single Chip 2X2 802.11 A/B/G/N MIMO MAC/BB/Radio, Data Sheet, Septembre 2016.

17. https://www.thethingsnetwork.org/docs/lorawan/duty-cycle.html. 